\title{
NORMAL-CONVEX EMBEDDINGS OF INVERSE SEMIGROUPS
}

\author{
by PEDRO V. SILVA
}

(Received 20 September, 1991)

Normal-convex embeddings are introduced for inverse semigroups, generalizing the group-theoretic concept, due to Papakyriakopoulos [4]. It is shown that every $E$-unitary inverse semigroup admits a normal-convex embedding into a semidirect product of a semilattice by a group, a stronger version of a result by $\mathrm{O}^{\prime}$ Carroll [3]. A general embedding result for inverse semigroups is also obtained.

1. Preliminaries. The general terminology and notation are those of Petrich [5].

Let $S$ be an inverse semigroup and let $R \subseteq S \times S$ be a relation on $S$. We denote by $R^{\#}$ the congruence on $S$ generated by $R$, that is, the transitive closure of $\{(a u b, a v b): a, b \in$ $S^{1}$ and $\left.(u, v) \in R \cup R^{-1}\right\}$. The natural projection $S \rightarrow S / R^{\#}$ is denoted by $\left(R^{\#}\right)^{\natural}$.

Let $\varphi: S \rightarrow T$ be a homomorphism of inverse semigroups and let $R$ be a relation on $S$. The relation

$$
R \varphi=\{(u \varphi, v \varphi):(u, v) \in R\}
$$

is said to be the relation on $T$ induced by $R$ and $\varphi$. It follows easily that

$$
R^{\#} \varphi \subseteq(R \varphi)^{\#} .
$$

If $\varphi$ is injective, we say that $\varphi$ is an embedding of inverse semigroups.

Now let $\varphi: S \rightarrow T$ be an embedding of inverse semigroups. We say that $\varphi$ is normal-convex if and only if, for every relation $R$ on $S$,

$$
(R \varphi)^{\#} \cap(S \times S) \varphi \subseteq R^{\#} \varphi .
$$

Note that, by (1.1), the inclusion $R^{\#} \varphi \subseteq(R \varphi)^{\#} \cap(S \times S) \varphi$ is always true. Also by (1.1), we know that $\varphi$ induces a unique homomorphism $\varphi_{R}: S / R^{\#} \rightarrow T /(R \varphi)^{\#}$ such that the canonical diagram

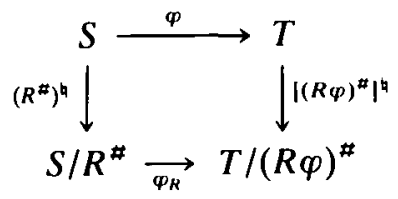

commutes. Now we have

Lemma 1.1. Let $\varphi: S \rightarrow T$ be an embedding of inverse semigroups. Then $\varphi$ is normal-convex if and only if $\varphi_{R}$ is injective for every relation $R$ on $S$.

Proof. Suppose that $\varphi$ is normal-convex and let $R$ be a relation on $S$. Let $a, b \in S$ be such that $\left(a R^{\#}\right)_{\varphi_{R}}=\left(b R^{\#}\right)_{\varphi_{R}}$. Since $(1.2)$ commutes, we have $(a \varphi)(R \varphi)^{\#}=(b \varphi)(R \varphi)^{\#}$. Hence $(a \varphi, b \varphi) \in(R \varphi)^{\#} \cap(S \times S) \varphi$. Since $\varphi$ is normal-convex, this yields $(a \varphi, b \varphi) \epsilon$ $R^{\#} \varphi$. Thus $a R^{\#}=b R^{\#}$ and so $\varphi_{R}$ is injective.

Conversely, suppose that $\varphi_{R}$ is injective for every relation $R$ on $S$. Suppose that $(a \varphi, b \varphi) \in(R \varphi)^{\#}$ for some $a, b \in S$. Since $(1.2)$ commutes, we have $\left(a R^{\#}\right)_{\varphi_{R}}=\left(b R^{\#}\right)_{\varphi_{R}}$, 
and since $\varphi_{R}$ is injective, $a R^{\#}=b R^{\#}$. Therefore $(a \varphi, b \varphi) \in R^{\#} \varphi$ and so $\varphi$ is normalconvex.

The following result shows that the class of normal-convex embeddings is closed under composition.

LeMma 1.2. Let $\varphi: S \rightarrow T$ and $\psi: T \rightarrow U$ be normal-convex embeddings of inverse semigroups. Then $\varphi \psi$ is a normal-convex embedding.

Proof. It is trivial that $\varphi \psi$ is an embedding. Now let $R$ be a relation on $S$. Since $(\varphi \psi)_{R}$ is uniquely defined, we certainly have $(\varphi \psi)_{R}=\varphi_{R} \psi_{R \varphi}$ and so $(\varphi \psi)_{R}$ is injective. Thus, by Lemma 1.1, $\varphi \psi$ is normal-convex.

The next result shows an application of the concept of normal-convex embedding.

Given a semigroup $S$ and a relation $R$ on $S$, the word problem for $R$ consists in finding an algorithm which determines, for every $a, b \in S$, whether or not $(a, b) \in R^{\#}$.

THEOREM 1.3. Let $\varphi: S \rightarrow T$ be a normal-convex embedding of inverse semigroups and let $R$ be a relation on $S$. Then the word problem for $R$ is solvable if the word problem for $R \varphi$ is solvable.

Proof. Suppose that the word problem for $R \varphi$ is solvable. Let $a, b \in S$. By Lemma $1.1, \varphi_{R}$ is injective and so $a R^{\#}=b R^{\#} \Leftrightarrow\left(a R^{\#}\right) \varphi_{R}=\left(b R^{\#}\right) \varphi_{R}$. Since (1.2) commutes, we have $\left(a R^{\#}\right)_{\varphi_{R}}=\left(b R^{\#}\right)_{\varphi_{R}} \Leftrightarrow(a \varphi)(R \varphi)^{\#}=(b \varphi)(R \varphi)^{\#}$. Since the word problem for $R \varphi$ is solvable, we can determine whether or not this latter equality holds, hence the word problem for $R$ is solvable and the theorem is proved.

Now let $S$ be an inverse semigroup with semilattice of idempotents $E(S)$. The least group congruence on $S$ is defined by

$$
(a, b) \in \sigma \Leftrightarrow \exists e \in E(S): a e=b e .
$$

We say that $S$ is $E$-unitary if

$$
\forall a \in S, \quad a \sigma=1 \Rightarrow a \in E(S) .
$$

Let $M$ denote an inverse monoid with least group congruence $\sigma$. Then $M$ is said to be $F$-inverse if every $\sigma$-class of $M$ has a maximal element under the natural partial order. It is well-known that every $F$-inverse monoid is $E$-unitary [5, §VII.5].

Let $G$ be a group and let $K$ be a semilattice. An action of $G$ on $K$ by left automorphisms is a map $G \times K \rightarrow K:(g, A) \mapsto g A$ such that, for every $g, h \in G$ and $A, B \in K$,

$$
\begin{aligned}
g(h A) & =(g h) A, \\
g(A B) & =(g A)(g B), \\
1 A & =A .
\end{aligned}
$$

It follows easily that, for every $g \in G$ and $A, B \in K$, we have

$$
A \leqslant B \Rightarrow g A \leqslant g B \text {. }
$$

The semidirect product of $K$ by $G$ induced by this action is the inverse semigroup $K \times G$ with the operation given by $(A, g)(B, h)=(A(g B), g h)$. When no ambiguity arises about the action, we shall denote this semigroup by $K \overline{\times} G$. 
Now suppose that $L$ is an ideal of $K$ such that $G L=K$. Then we say that $(G, K, L)$ is a strong McAlister triple and

$$
P(G, K, L)=\left\{(A, g) \in L \times G: g^{-1} A \in L\right\}
$$

is an inverse subsemigroup of $K \overline{\times} G[\mathbf{1}]$.

Lemma 1.4 [1]. Let $M$ be an inverse monoid. Then $M$ is $F$-inverse if and only if $M \simeq P(G, K, L)$ for some strong McAlister triple $(G, K, L)$ such that $L$ has a unity.

Let $S$ be an inverse semigroup and let $\tau$ be a congruence on $S$. We say that $\tau$ is idempotent-pure if, for every $(a, b) \in \tau$,

$$
a \in E(S) \Rightarrow b \in E(S) \text {. }
$$

We say that $\tau$ is idempotent-separating if, for every $(a, b) \in \tau$,

$$
a \in E(S) \Rightarrow b \notin E(S) \text {. }
$$

Finally, an inverse semigroup $S$ is said to be quasi-free if $T \simeq F / \tau$ for some free inverse semigroup $F$ and some idempotent-pure congruence $\tau$ on $F$.

Lemma 1.5 [2]. Let $S$ be a quasi-free inverse semigroup. Then $S \simeq P(G, K, L)$ for some strong McAlister triple $(G, K, L)$ with $G$ free.

2. Strong McAlister triples. In this section we show that, for every strong McAlister triple $(G, K, L)$, there exists a canonical embedding of $P(G, K, L)$ into a semidirect product of a semilattice by a group.

TheOREm 2.1. Let $(G, K, L)$ be a strong McAlister triple. Then the inclusion map $\varphi: P(G, K, L) \rightarrow K \overline{\times} G$ is normal-convex.

Proof. Let $S=P(G, K, L)$ and let $T=K \bar{x} G$. Let $R$ be a relation on $S$, say $R=\left\{\left(\left(A_{i}, g_{i}\right),\left(B_{i}, h_{i}\right)\right): i \in I\right\}$. Without loss of generality, we can assume that $R$ is symmetric. Let $(U, u),(V, v) \in S$ be such that $(U, u)(R \varphi)^{\#}=(V, v)(R \varphi)^{\#}$. We want to prove that $(U, u) R^{*}=(V, v) R^{*}$. Since $R$ is symmetric, we know that there exist $\left(W_{0}, w_{0}\right), \ldots,\left(W_{n}, w_{n}\right) \in T$ such that

$$
\begin{gathered}
\left(W_{0}, w_{0}\right)=(U, u) \\
\left(W_{n}, w_{n}\right)=(V, v) \\
\forall j \in\{1, \ldots, n\} \exists\left(P_{j}, p_{j}\right),\left(Q_{j}, q_{j}\right) \in T \exists i_{j} \in I: \\
\left(W_{j-1}, w_{j-1}\right)=\left(P_{j}, p_{j}\right)\left(A_{i_{j}}, g_{i j}\right)\left(Q_{j}, q_{j}\right)
\end{gathered}
$$

and

$$
\left(W_{j}, w_{j}\right)=\left(P_{j}, p_{j}\right)\left(B_{i}, h_{i_{i}}\right)\left(Q_{j}, q_{j}\right)
$$

Now we show that, for every $m \in\{0, \ldots, n\}$,

$$
\begin{aligned}
& \exists P_{m}^{\prime}, Q_{m}^{\prime}, W_{m}^{\prime} \in L: \\
& \quad\left(W_{m}^{\prime}, w_{m}\right) \in S, \\
& \left(W_{m}^{\prime}, w_{m}\right) R^{\#}=(U, u) R^{\#}, \\
& \left(W_{m}^{\prime}, w_{m}\right)=\left(P_{m}^{\prime}, 1\right)\left(W_{m}, w_{m}\right)\left(Q_{m}^{\prime}, 1\right) .
\end{aligned}
$$


We use induction on $m$. Defining $P_{0}^{\prime}=U, Q_{0}^{\prime}=u^{-1} U$ and $W_{0}^{\prime}=U$, we see that (2.1) holds for $m=0$.

Now suppose that (2.1) holds for $m=j-1$, with $j \in\{1, \ldots, n\}$. Then

$$
\begin{aligned}
\left(W_{j-1}^{\prime}, w_{j-1}\right) & =\left(W_{j-1}^{\prime}, 1\right)\left(W_{j-1}^{\prime}, w_{j-1}\right)\left(w_{j-1}^{-1} W_{j-1}^{\prime}, 1\right) \\
& =\left(W_{j-1}^{\prime}, 1\right)\left(P_{j-1}^{\prime}, 1\right)\left(W_{j-1}, w_{j-1}\right)\left(Q_{j-1}^{\prime}, 1\right)\left(w_{j-1}^{-1} W_{j-1}^{\prime}, 1\right) \\
& =\left(W_{j-1}^{\prime}, 1\right)\left(P_{j-1}^{\prime}, 1\right)\left(P_{j}, p_{j}\right)\left(A_{i j}, g_{i j}\right)\left(Q_{j}, q_{j}\right)\left(Q_{j-1}^{\prime}, 1\right)\left(w_{j-1}^{-1} W_{j-1}^{\prime}, 1\right) .
\end{aligned}
$$

It is clear that

and so

$$
W_{j-1}^{\prime} \leqslant P_{j-1}^{\prime} P_{j}
$$

Similarly,

$$
\left(W_{j-1}^{\prime}, 1\right)\left(P_{j-1}^{\prime}, 1\right)\left(P_{j}, p_{j}\right)=\left(W_{j-1}^{\prime}, p_{j}\right)
$$

and so

$$
W_{j \sim 1}^{\prime} \leqslant\left(p_{j} g_{i_{j}} Q_{j}\right)\left(p_{i} g_{i j} q_{j} Q_{i-1}^{\prime}\right)
$$

Hence

$$
g_{i_{j}}^{-1} p_{j}^{-1} W_{j-1}^{\prime} \leqslant Q_{j}\left(q_{j} Q_{j-1}^{\prime}\right) .
$$

Thus

$$
\left(Q_{j}, q_{j}\right)\left(Q_{j-1}^{\prime}, 1\right)\left(w_{j-1}^{-1} W_{j-1}^{\prime}, 1\right)=\left(g_{i_{j}}^{-1} p_{j}^{-1} W_{j-1}^{\prime}, q_{j}\right) .
$$

$$
\left(W_{j-1}^{\prime}, w_{j-1}\right)=\left(W_{j-1}^{\prime}, p_{j}\right)\left(A_{i_{i}}, g_{i_{j}}\right)\left(g_{i_{j}}^{-1} p_{j}^{-1} W_{j-1}^{\prime}, q_{j}\right) \text {. }
$$

Since $W_{j-1}^{\prime} \leqslant p_{j} A_{i_{i}}$, we have $p_{j}^{-1} W_{j-1}^{\prime} \leqslant A_{i_{i}} \in L$. But $L$ is an ideal of $K$ and so $p_{j}^{-1} W_{j-1}^{\prime} \in L$. Since $W_{j-1}^{\prime} \in L$, we obtain $\left(W_{j-1}^{\prime}, p_{j}\right) \in S$. Similarly, we have $g_{i_{j}}^{-1} p_{j}^{-1} W_{j-1}^{\prime} \leqslant$ $g_{i_{j}}^{-1} p_{j}^{-1}\left(p_{j} A_{i_{j}}\right)=g_{i_{j}}^{-1} A_{i_{j}} \in L$, and $q_{j}^{-1} g_{i_{j}}^{-1} p_{j}^{-1} W_{j-1}^{\prime}=w_{j-1}^{-1} W_{j-1}^{\prime} \in L$. Hence

$$
\left(g_{i_{j}}^{-1} p_{j}^{-1} W_{j-1}^{\prime}, q_{j}\right) \in S \text {. }
$$

Let $P_{j}^{\prime}=W_{j-1}^{\prime}, \quad Q_{j}^{\prime}=w_{j-1}^{-1} W_{j-1}^{\prime}$ and $W_{j}^{\prime}=W_{j-1}^{\prime}\left(p_{j} B_{i_{i}}\right)\left(w_{j} w_{j-1}^{-1} W_{j-1}^{\prime}\right)$. Obviously, $P_{j}^{\prime}, Q_{j}^{\prime} \in L$ and since $L$ is an ideal of $K$, we have $W_{j}^{\prime} \in L$ as well. We have $\left(W_{j}^{\prime}, w_{j}\right)=\left(W_{j-1}^{\prime}, p_{j}\right)\left(B_{i_{j}}, h_{i_{j}}\right)\left(g_{i_{j}}^{-1} p_{j}^{-1} W_{j-1}^{\prime}, q_{j}\right)$, that is, $\left(W_{j}^{\prime}, w_{j}\right)$ is a product of elements of $S$. Therefore $\left(W_{j}^{\prime}, w_{j}\right) \in S$. Moreover,

$$
\begin{aligned}
\left(W_{j}^{\prime}, w_{j}\right) R^{\#} & =\left[\left(W_{j-1}^{\prime}, p_{j}\right)\left(B_{i_{i}}, h_{i_{i}}\right)\left(g_{i_{j}}^{-1} p_{j}^{-1} W_{j-1}^{\prime}, q_{j}\right)\right] R^{\#} \\
& =\left[\left(W_{j-1}^{\prime}, p_{j}\right)\left(A_{i_{i}}, g_{i_{j}}\right)\left(g_{i_{j}}^{-1} p_{j}^{-1} W_{j-1}^{\prime}, q_{j}\right)\right] R^{\#}=\left(W_{j-1}^{\prime}, w_{j-1}\right) R^{\#}=(U, u) R^{\#} .
\end{aligned}
$$

It follows from (2.2) that $\left(W_{j-1}^{\prime}, p_{j}\right)=\left(W_{j-1}^{\prime}, 1\right)\left(P_{j}, p_{j}\right)$. Similarly, (2.3) yields $\left(g_{i_{j}}^{-1} p_{j}^{-1} W_{j-1}^{\prime}, q_{j}\right)=\left(Q_{j}, q_{j}\right)\left(w_{j-1}^{-1} W_{j-1}^{\prime}, 1\right)$. Hence

$$
\begin{aligned}
\left(W_{j}^{\prime}, w_{j}\right) & =\left(W_{j-1}^{\prime}, p_{j}\right)\left(B_{i j}, h_{i_{i}}\right)\left(g_{i_{j}}^{-1} p_{j}^{-1} W_{j-1}^{\prime}, q_{j}\right) \\
& =\left(W_{j-1}^{\prime}, 1\right)\left(P_{j}, p_{j}\right)\left(B_{i_{j}}, h_{i_{j}}\right)\left(Q_{j}, q_{j}\right)\left(w_{j-1}^{-1} W_{j-1}^{\prime}, 1\right)=\left(P_{j}^{\prime}, 1\right)\left(W_{j}, w_{j}\right)\left(Q_{j}^{\prime}, 1\right)
\end{aligned}
$$

and so (2.1) holds for $m=j$.

Thus (2.1) holds for every $m \in\{0, \ldots, n\}$. In particular, we have $\left(W_{n}^{\prime}, v\right) R^{\#}=$ $\left(W_{n}^{\prime}, w_{n}\right) R^{\#}=(U, u) R^{\#} \quad$ and $\quad\left(W_{n}^{\prime}, v\right)=\left(P_{n}^{\prime}, 1\right)\left(W_{n}, w_{n}\right)\left(Q_{n}^{\prime}, 1\right)=\left(P_{n}^{\prime}, 1\right)(V, v)\left(Q_{n}^{\prime}, 1\right)$. Therefore $W_{n}^{\prime} \leqslant V$ and so $\left(W_{n}^{\prime}, v\right)=\left(W_{n}^{\prime}, 1\right)(V, v)$. It follows that $(U, u) R^{\#}=$ $\left(W_{n}^{\prime}, 1\right) R^{\#}(V, v) R^{\#}$ and so $(U, u) R^{\#} \leqslant(V, v) R^{\#}$. Similarly, we obtain $(V, v) R^{\#} \leqslant$ $(U, u) R^{\#}$ and so $(U, u) R^{\#}=(V, v) R^{\#}$. Thus $\varphi$ is normal-convex.

Now, Lemma 1.5 and Theorem 2.1 immediately yield 
COROLlaRY 2.2. Every quasi-free inverse semigroup admits a normal-convex embedding into a semidirect product of a semilattice by a free group.

Since every free inverse semigroup is quasi-free, we also obtain

COROLlaRY 2.3. Every free inverse semigroup admits a normal-convex embedding into a semidirect product of a semilattice by a free group.

3. $E$-unitary inverse semigroups. In this section we prove that every $E$-unitary inverse semigroup admits a normal-convex embedding into a semidirect product of a semilattice by a group.

Let $S$ be an $E$-unitary inverse semigroup. Let $M(S)=\{\varnothing \notin A \subseteq S: E(S) . A \subseteq A \subseteq a \sigma$ for some $a \in S\}$ with the operation described by $A B=\{a b: a \in A$ and $b \in B\}$. The following result is due to O'Carroll.

Lemma 3.1 [3]. Let $S$ be an E-unitary inverse semigroup. Then $M(S)$ is an $F$-inverse monoid and the map $\varphi: S \rightarrow M(S): s \mapsto\{t \in S: t \leqslant s\}$ is an embedding. Moreover, if $\sigma_{S}$ and $\sigma_{M(S)}$ denote respectively the least group congruences of $S$ and $M(S)$, then $\sigma_{M(S)} \cap$ $(S \times S) \varphi=\sigma_{S} \varphi$.

We prove that this embedding is in fact normal-convex.

Lemma 3.2. Let $S$ be an E-unitary inverse semigroup. Then the embedding $\varphi: S \rightarrow$ $M(S): s \mapsto\{t \in S: t \leqslant s\}$ is normal-convex.

Proof. Let $R$ be a relation on $S$. Without loss of generality, we can assume that $R$ is symmetric. Let $a, b \in S$ be such that $(a \varphi, b \varphi) \in(R \varphi)^{\#}$. We want to prove that $(a, b) \in R^{\#}$.

Since $(a \varphi, b \varphi) \in(R \varphi)^{\#}$, there exist $W_{0}, \ldots, W_{n} \in M(S)$ such that

$$
\begin{gathered}
W_{0}=a \varphi ; \\
W_{n}=b \varphi ; \\
\forall i \in\{1, \ldots, n\} \exists P_{i}, Q_{i} \in M(S) \exists\left(u_{i}, v_{i}\right) \in R \\
W_{i-1}=P_{i}\left(u_{i} \varphi\right) Q_{i} \text { and } W_{i}=P_{i}\left(v_{i} \varphi\right) Q_{i} .
\end{gathered}
$$

We prove the following result. Let $z \in S$ and $C, D \in M(S)$ be such that $C(z \varphi) D \in S \varphi$. Then

$$
\exists c, d \in S: c \varphi \subseteq C, \quad d \varphi \subseteq D \text { and }(c z d) \varphi=C(z \varphi) D .
$$

Since $C(z \varphi) D \in S \varphi$, there exists some $w \in S$ such that $C(z \varphi) D=w \varphi$. Since $w \in w \varphi$, there exist $c \in C, z^{\prime} \in z \varphi$ and $d \in D$ such that $c z^{\prime} d=w$. Since $c \varphi \subseteq C, z^{\prime} \varphi \subseteq z \varphi$ and $d \varphi \subseteq D$, we obtain $w \varphi=\left(c z^{\prime} d\right) \varphi=(c \varphi)\left(z^{\prime} \varphi\right)(d \varphi) \subseteq(c \varphi)(z \varphi)(d \varphi) \subseteq C(z \varphi) D=w \varphi$. Therefore $(c z d) \varphi=C(z \varphi) D$ and (3.1) holds.

Since $S$ is $E$-unitary, it is clear that

$$
\forall A \in M(S), \quad A A^{-1} \subseteq 1 \sigma \subseteq E(S) .
$$

Now we show that, for every $j \in\{0, \ldots, n\}$

$$
\exists w_{j} \in S: w_{j} \varphi \subseteq W_{j} \text { and }\left(a, w_{j}\right) \in R^{*} .
$$


Let $w_{0}=a$. It follows that (3.3) holds for $j=0$.

Now suppose that (3.3) holds for $j=i-1$, with $i>0$. Then $w_{i-1} \varphi \subseteq W_{i-1}$ and so, since $S$ is inverse, $w_{i-1} \varphi \subseteq W_{i-1} W_{i-1}^{-1}\left(w_{i-1} \varphi\right)$. By (3.2), we also have $W_{i-1} W_{i-1}^{-1}\left(w_{i-1} \varphi\right) \subseteq$ $w_{i-1} \varphi$. Hence $w_{i-1} \varphi=W_{i-1} W_{i-1}^{-1}\left(w_{i-1} \varphi\right)=P_{i}\left(u_{i} \varphi\right) Q_{i} W_{i-1}^{-1}\left(w_{i-1} \varphi\right)$. Now we can apply (3.1) with $z=u_{i}, C=P_{i}$ and $D=Q_{i} W_{i-1}^{-1}\left(w_{i-1} \varphi\right)$. Hence there exist $p_{i}, q_{i} \in S$ such that $p_{i} \varphi \subseteq P_{i}, q_{i} \varphi \subseteq Q_{i} W_{i-1}^{-1}\left(w_{i-1} \varphi\right)$ and $\left(p_{i} u_{i} q_{i}\right) \varphi=P_{i}\left(u_{i} \varphi\right) Q_{i} W_{i-1}^{-1}\left(w_{i-1} \varphi\right)=w_{i-1} \varphi$. We define $w_{i}=p_{i} v_{i} q_{i}$. Now $w_{i} \varphi=\left(p_{i} \varphi\right)\left(v_{i} \varphi\right)\left(q_{i} \varphi\right) \subseteq P_{i}\left(v_{i} \varphi\right) Q_{i} W_{i-1}^{-1}\left(w_{i-1} \varphi\right)=W_{i} W_{i-1}^{-1}\left(w_{i-1} \varphi\right) \subseteq$ $W_{i} W_{i-1}^{-1} W_{i-1}$ and so, by (3.2), we have $w_{i} \varphi \subseteq W_{i} . E(S)$. For every $s \in S$ and $e \in E(S)$, we have $a e=a e a^{-1} a$, and hence $W_{i} . E(S) \subseteq E(S) . W_{i}$. Therefore $w_{i} \varphi \subseteq W_{i} . E(S) \subseteq$ $E(S) . W_{i} \subseteq W_{i}$. Moreover, $w_{i} R^{\#}=\left(p_{i} v_{i} q_{i}\right) R^{\#}=\left(p_{i} u_{i} q_{i}\right) R^{\#}=w_{i-1} R^{\#}=a R^{\#}$ and so (3.3) holds for $j=i$. Thus (3.3) holds for every $j \in\{0, \ldots, n\}$.

In particular, $w_{n} \varphi \subseteq W_{n}=b \varphi$ and $\left(a, w_{n}\right) \in R^{\#}$. Hence $w_{n} \leqslant b$ and $a R^{\#}=w_{n} R^{\#} \leqslant$ $b R^{\#}$. Similarly, we prove that $b R^{\#} \leqslant a R^{\#}$. Thus $(a, b) \in R^{\#}$ and the lemma is proved.

Now we obtain

TheOREM 3.3. Every E-unitary inverse semigroup admits a normal-convex embedding into a semidirect product of a semilattice by a group.

Proof. Let $S$ be an $E$-unitary inverse semigroup. By Lemma 3.2, the embedding $\varphi: S \rightarrow M(S): s \mapsto\{t \in S: t \leqslant s\}$ is normal-convex. By Lemma $3.1, M(S)$ is $F$-inverse and so, by Lemma 1.4 and Theorem 2.1 , there exists a normal-convex embedding $\psi: M(S) \rightarrow$ $P$, where $P$ is a semidirect product of a semilattice by a group. By Lemma 1.2 , the composition $\varphi \psi: S \rightarrow P$ is a normal-convex embedding and the theorem is proved.

4. Inverse semigroups. The results of Section 2 can be used to obtain a general embedding result on inverse semigroups. We shall make use of the following result on quasi-free covers, due to Munn and Reilly.

LemMA 4.1 [2]. Let $S$ be an inverse semigroup. Then there exists a quasi-free inverse semigroup $F$ and an idempotent-separating congruence $\tau$ on $F$ such that $S \simeq F / \tau$.

Now we have

THEOREM 4.2. Every inverse semigroup admits a normal-convex embedding into an idempotent-separating homomorphic image of a semidirect product of a semilattice by $a$ free group.

Proof. Let $S$ be an inverse semigroup. By Lemma 4.1, we can assume that $S=F / \tau$, with $F$ quasi-free and $\tau$ idempotent-separating. By Lemma 1.5, we can assume that $F=P(G, K, L)$ for some strong McAlister triple $(G, K, L)$, with $G$ free. By Theorem 2.1, the inclusion $\varphi: F \rightarrow K \overline{\times} G$ is normal-convex. Therefore, by Lemma 1.1 , the induced map $\psi: F / \tau \rightarrow(K \overline{\times} G) /(\tau \varphi)^{\#}$ defined by $(a \tau) \psi=a(\tau \varphi)^{\#}$ is injective. We must prove that $\psi$ is normal-convex and $(\tau \varphi)^{\#}$ is idempotent-separating.

First we prove that $\psi$ is normal-convex. Let $T=(K \overline{\times} G) /(\tau \varphi)^{\#}$. Let $R$ be a relation on $S$. We want to show that $(R \psi)^{\#} \cap(S \times S) \psi \subseteq R^{\#} \psi$.

Let $\mu$ be the congruence on $F$ such that $\mu / \tau=R^{\#}$. It follows that, for every $a, b \in F$, $(a, b) \in \mu$ if and only if $(a \tau, b \tau) \in R^{\#}$. We prove that

$$
(R \psi)^{\#} \subseteq(\mu \varphi)^{\#} /(\tau \varphi)^{\#} .
$$

Since $\tau \subseteq \mu$, we have $\tau \varphi \subseteq \mu \varphi$ and so $(\tau \varphi)^{\#} \subseteq(\mu \varphi)^{\#}$. Hence $(\mu \varphi)^{\#} /(\tau \varphi)^{\#}$ is a congruence on $T$ and we only need to show that $R \psi \subseteq(\mu \varphi)^{\#} /(\tau \varphi)^{\#}$. Let $a, b \in F$ be such 
that $(a \tau, b \tau) \in R$. Then $(a \tau, b \tau) \in R^{\#}$ and so, by definition of $\mu$, we have $(a, b) \in \mu$. Hence $(a \varphi, b \varphi) \in \mu \varphi \subseteq(\mu \varphi)^{\#}$. Therefore $\left(a \varphi(\tau \varphi)^{\#}, b \varphi(\tau \varphi)^{\#}\right) \in(\mu \varphi)^{\#} /(\tau \varphi)^{\#}$, that is, $((a \tau) \psi,(b \tau) \psi) \in(\mu \varphi)^{\#} /(\tau \varphi)^{\#}$. Hence (4.1) holds.

Now suppose that $a, b \in F$ and $((a \tau) \psi,(b \tau) \psi) \in(R \psi)^{\#}$. Then, by (4.1), we have $((a \tau) \psi,(b \tau) \psi) \in(\mu \varphi)^{\#} /(\tau \varphi)^{\#}$. Hence $\left(a \varphi(\tau \varphi)^{\#}, b \varphi(\tau \varphi)^{\#}\right) \in(\mu \varphi)^{\#} /(\tau \varphi)^{\#}$ and so $(a \varphi, b \varphi) \in(\mu \varphi)^{\#}$. Since $\varphi$ is normal-convex and $\mu$ is a congruence on $F$, we have $(\mu \varphi)^{\#} \cap(F \times F) \varphi \subseteq \mu \varphi$. Hence $(a \varphi, b \varphi) \in \mu \varphi$ and so $(a, b) \in \mu$ and $(a \tau, b \tau) \in R^{\#}$. Therefore $((a \tau) \psi,(b \tau) \psi) \in R^{\#} \psi$ and so $\psi$ is normal-convex.

Now we prove that $(\tau \varphi)^{\#}$ is idempotent-separating. Obviously, $E(K \overline{\times} G)=$ $\{(A, 1): A \in K\}$. Suppose that $A, B \in K$ are such that $(A, 1)(\tau \varphi)^{\#}=(B, 1)(\tau \varphi)^{\#}$. Since $G L=K$, there exists $g \in G$ and $C \in L$ such that $g C=A$. Hence $g^{-1} A=C \in L$ and we have

$$
\begin{aligned}
\left(g^{-1} A, 1\right)(\tau \varphi)^{\#} & =\left[\left(g^{-1} A, g^{-1}\right)(A, 1)(A, g)\right](\tau \varphi)^{\#}=\left[\left(g^{-1} A, g^{-1}\right)(B, 1)(A, g)\right](\tau \varphi)^{\#} \\
& =\left(\left(g^{-1} A\right)\left(g^{-1} B\right), 1\right)(\tau \varphi)^{\#} .
\end{aligned}
$$

Since $\left(g^{-1} A\right)\left(g^{-1} B\right) \leqslant g^{-1} A \in L$ and $L$ is an ideal of $K$, we have $\left(g^{-1} A\right)\left(g^{-1} B\right) \in L$. Hence $\left(g^{-1} A, 1\right),\left(\left(g^{-1} A\right)\left(g^{-1} B\right), 1\right) \in F$. But

$$
\left[\left(g^{-1} A, 1\right) \tau\right] \psi=\left(g^{-1} A, 1\right)(\tau \varphi)^{\#}=\left(\left(g^{-1} A\right)\left(g^{-1} B\right), 1\right)(\tau \varphi)^{\#}=\left[\left(\left(g^{-1} A\right)\left(g^{-1} B\right), 1\right) \tau\right] \psi
$$

and so, since $\psi$ is injective, $\left(g^{-1} A, 1\right) \tau=\left(\left(g^{-1} A\right)\left(g^{-1} B\right), 1\right) \tau$. Since $\tau$ is idempotentseparating, we obtain $\left(g^{-1} A, 1\right)=\left(\left(g^{-1} A\right)\left(g^{-1} B\right), 1\right)$, that is, $g^{-1} A=\left(g^{-1} A\right)\left(g^{-1} B\right)$. Hence $A=A B$ and $A \leqslant B$. Similarly, we obtain $B \leqslant A$ and so $A=B$. Thus $(A, 1)=(B, 1)$ and $(\tau \varphi)^{\#}$ is idempotent-separating.

ACKNowledgements. This work has been carried out while I held a research grant from the Calouste Gulbenkian Foundation, which I thank. I am also grateful to Prof. W. D. Munn for all the help and advice provided.

\section{REFERENCES}

1. D. B. McAlister, Groups, semilattices and inverse semigroups II, Trans. Amer. Math. Soc. 196 (1974), 351-370.

2. W. D. Munn and N. R. Reilly, E-unitary congruences on inverse semigroups, Glasgow Math. J. 17 (1976), 57-75.

3. L. O'Carroll, Reduced inverse and partially ordered semigroups, J. London Math. Soc. (2)9 (1974), 293-301.

4. C. D. Papakyriakopoulos, A new proof for the invariance of the homology groups of a complex, Bull. Soc. Math. Grèce 22 (1943), 1-154.

5. M. Petrich, Inverse semigroups, (Wiley, 1984).

Departamento de Matemática

UnIVERSIDADE DO PORTO

4000 PORTO

Portugal
Department of Mathematics

UNIVERSITY GARDENS

GLASGOW G12 8QW 